\title{
Future Prediction of Cardiovascular Disease Using Deep Learning Technique
}

\author{
Indhumathi.M ${ }^{\mathrm{a}, 1}$ and Dr.V.D.AmbethKumar ${ }^{\mathrm{b}}$ \\ ${ }^{\mathrm{a}, 1}$ PG Scholar, Department of CSE, Panimalar Engineering College, Chennai, India \\ bProfessor, Department of CSE, Panimalar Engineering College, Chennai, India
}

\begin{abstract}
Cardiovascular disease is the one of the most leading causes of death. Based on symptoms and risk factors the diagnosis of heart can be done. Predicting the cardiovascular disease in the early stage can save the human being. There is no complete cure which reduces the risk of CVD.Deep learning technique has been used to predict the CVD in a prior stage. Based on the symptoms and risk factors, the CVD has been classified into four types such as No heart disease and no symptoms, Structural Heart Disease without symptoms, Structural Heart Disease with Symptoms and the risk factor for Heart failure are "High blood pressure, high cholesterol, genetic, diabetes, obesity is the major risk factors" to identify the cardiovascular disease and current technique is used to control the risks. To manage all the risk factors Electrocardiography (ECG) method is used to manipulate based on particular situation.
\end{abstract}

Keywords: CVD, Healthcare, risk factors.

\section{Introduction}

An estimation done by World Health Organisation (WHO) in the year 2016 for the CVD patients is nearly about 17.9 million people. It is nearly $31 \%$. Because of CVD the huge death occurs. The group of disorder of heart and blood vessels includes coronary heart disease, rheumatic heart disease and other conditions. Under the age of 70 years, one third of the death occurs because of heart attack and stroke. In an earlier stage it is very difficult to predict the CVD affected patients. Based on the symptoms and risk factors the CVD affected patients has been found out. Some of the risk factors like "high blood pressure, Diabetes, High blood Cholesterol, genetic and obesity. Some of the symptoms like pain in chest, shortness of breath, cold sweat and nausea and fatigue. Coronary heart disease ,High blood pressure, Cardiac Arrest, Heart failure and Arrhythmia, Peripheral artery disease and stroke are the some of the types of heart disease.

${ }^{1}$ Indhumathi.M, Student, Department of CSE, Panimalar Engineering College, Chennai, India. E-mail: mindu1910@gmail.com 


\section{Related work}

From the sudden increase of blood pressure with the development of acute decompensate heart failure (ADHF). Out of 3239, 113 patients were analyzed and produced the composite endpoint group and non-composite endpoint group. The cut-off value for composite endpoint was 8.65. Need more clinical study to confirm this result[1]. To find the correlation between TG/HDL-Cholesterol ratio with cardiac risk factors. Chi-square test was computed to check relation of cardio metabolic risk factors like hypertension, diabetes and obesity with TG/HDL-C ratio[2]. The best predictor in XG Boost model. They has been developed a highly precise prediction model for future hypertension [3]. For maintaining normal cardiac and metabolic status Natriuretic Peptides (NP) are important and have been used to predicts cardiovascular events. The three bioactive counterparts such as NTproANP, NTproBNP and NTproCNP were measured randomly in 348 samples. Oppositely extract relation were found among plasma NTproBNP or NTproANP[4].Administrative database has been used and the data has been divided into derivation and validation samples. Two-third has been used for derivation and remaining has been used for validation [5]. The epigenetic mechanism lies between cancer and cardiovascular disease and a small importance to burgeoning field of cardio-oncology[6].To assess the utility of Computed Tomography (CT) based abdominal aortic calcification for the prediction of LVDD and prognosis of asymptomatic pre-dialysis CKD patients[7]. Without using CT, MRI or other diagnostic equipment they predict the concomitant development of macroangiopathy in diabetic in a single clinical has been evaluated. The major limitation of this study is the author may have missed a macroangiopathydiagnosis[8]. For analyzing medical images the deep learning algorithm [10-32], in specific CNN is the most popular one. In DL the survey has taken from classification, segmentation and object detection[9].

\section{System Architecture}

Fig 1 represents the collection of patient's detail, in which it contains the blood pressure, cholesterol, genetic, diabetes and obesity, etc. After the collection of dataset, pre-processing has been done. Data pre-processing is transforming the raw data into an understandable format. After the data pre-processing, the data cleaning has been done. Data cleaning is the process of process of removing incorrect or duplicate data within the dataset.

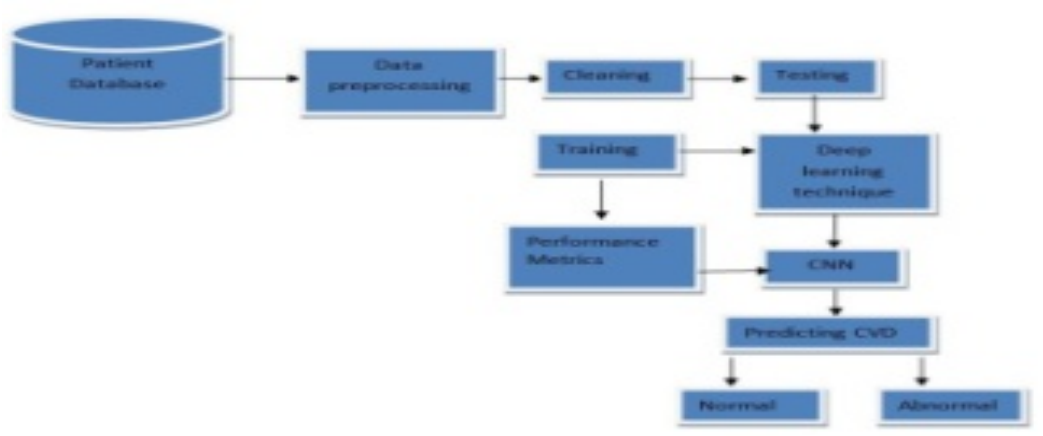

Figure 1.Recognize Cardiovascular Disease using Deep Learning Technique 
Training the models is said to be the data which should be trained and it is the initial set of data which is used to help a program understand how to apply deep learning techniques and produce sophisticated results. Testing the models is used to fit the model and testing data to test it and the generated models are to predict the results unknown which is said to be test set. The performance will be measured by using the test data only. The results will be collected for the two techniques which is used under the concept of convolution neural network and it will be compared to predict cardiovascular disease.

\section{Dataflow Diagrams}

\subsection{Level 0}

Collect a datasets from health care. Process the collected dataset and finding cardiovascular disease affected patients.

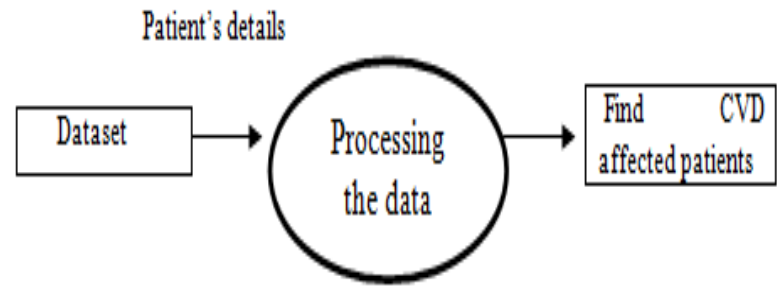

Figure 2.Level 0 DFD Diagram

\subsection{Level 1}

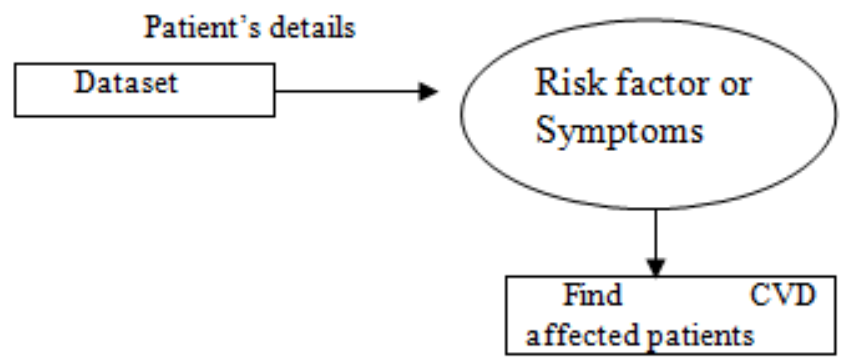

Figure 3. Level 1 DFD Diagram 


\section{Conclusion}

In this paper, different attributes have been used and we can easily find the cardiovascular disease patients by analysing all the attributes. If the patient level goes abnormal, it makes sure to intimate the medical practitioner and the cardiovascular disease affected patients will be identified and prevented easily.

\section{References}

[1] Takahide Sano,Shunsuke Kiuchi,Shinji Hisatake,et al, Cardio-ankle vascular index predicts the 1-year prognosis of heart failure patients categorized in clinical scenario 1 ,Heart and vessels,May2020

[2] QuratulAin,NaveedAsif,AmirAlam,et al, Triglycerides-to-HDLC Ratio as a Marker of Cardiac Disease and Vascular Risk Factors in Adults, Department of Chemical Pathology \& Endocrinology, Armed Forces Institute of Pathology (AFIP),vol 29(11)PP:1034-7,July 2019

[3] Hiroshi Kanega,KenjiSuzuki,Kyohei, et al, Highly precise risk prediction model for new-onset hypertension using artificial intelligence techniques, wileyonlinelibrary, 2019

[4] Timothy C. R. Prickett, Janet K. Spittlehouse, Allison L. Miller, YusmiatiLiau,et al, Contrasting signals of cardiovascular health among natriuretic peptides in subjects without heart disease ,scientific research,august 2019

[5] Husam Abdel-qadir,PaaladineshThavendiranathan,Peter C Austin,Douglas S Lee,et al, Development and validation of a multivariable prediction model for major adverse cardiovascular events after early stage breast cancer: a population-based cohort study, European society of cardiology,vol 40,PP 39133920,2019

[6] AbdalrahmanZarzour, Ha Won Kim, Neal L. Weintraub, Epigenetic Regulation of vascular disease ,ATVB in Focus Metabolic and oxidativestress,april 12019

[7] Kenji Furusawa,KyosukeTakeshita, Susumu Suzuki,et al, Assessment of abdominal aortic calcification by computed tomography for prediction of latent left ventricular stiffness and future cardiovascular risk in pre-dialysis patients with chronic kidney disease: A single center cross-sectional study ,International journal of medical sciences, vol.16,2019

[8] KiyohikoTakahashi,MidoriDannoura, et al , The association of cardio-Ankle vascular Index and AnkleBrachial index with Macroangiopathyin patiets with Type 2 Diabetes Mellitus, The official journal of the japan Atherosclerosis society and the Asian Pacific society of Atherosclerosis and vascular Diseases,vol.26,PP616-623,2019

[9] Geert Litjens* , ThijsKooi, BabakEhteshamiBejnordi, Arnaud ArindraAdiyosoSetio, Francesco Ciompi, Mohsen Ghafoorian, Jeroen A.W.M. van der Laak, Bram van Ginneken, Clara I. Sánchez”A survey on deep learning in medical image analysis",Medical image analysis,2017

[10] S.Hema Kumar, J.UdayKiran, V.D.AKumar, G.Saranya, Ramalakshmi V, "Effective Online Medical Appointment System", International Journal of Scientific \& Technology Research, Volume 8, Issue 09, September 2019, Pages $803-805$.

[11] Ambeth Kumar.V.D , Dr.M.Ramakrishnan, V.D.Ashok Kumar and Dr.S.Malathi (2015).Performance Improvement using an Automation System for Recognition of Multiple Parametric Features based on Human Footprint. kuwait journal of science, Vol 42, No 1 (2015), pp:109-132.

[12] Ambeth Kumar.V.D, V.D.Ashok Kumar, S.Malathi, K.Vengatesan, M.Ramakrishnan.Facial Recognition System For Suspect Identification Using A Surveillance Camera. Pattern Recognition and Image Analysis (Springer), Volume 28, Issue 3, pp 410-420, 2018. (DOI: $10.1134 / \mathrm{S} 1054661818030136)$

[13] V.D.Ambeth Kumar and M.Ramakrishan. Employment Of Footprint Recognition System. in the month of December for Indian Journal of Computer Science and Engineering (IJCSE) Vol. 3 No.6 Dec 2013

[14] Ramya,T.,Dr.Malathi,S.,ratheeksha,G.R. and Dr.V.D.Ambeth Kumar (2014). Personalized authentication procedure for restricted web service access in mobile phones.Applications of Digital Information and Web Technologies (ICADIWT), 2014, Page(s):69 - 74, Bangalore, India (ISBN:978-14799-2258-1)

[15] V.D.Ambeth Kumar (2018), “ NOVEL WIRELESS SENSING SYSTEM FOR THE WELFARE OF SEWER LABORERS”, Healthcare Technology Letters (IET) Volume 5, Issue 4, p. 107 -112. 
[16] V.D.Ambeth Kumar, G.Saranya , D.Elangovan, V.RahulChiranjeevi, V.D.Ashok Kumar, "IOT Based Smart Museum Using Wearable Device”, Lecture Notes in Networks and Systems, Vol.55, pp: 33-42, 2018

[17] V.D.Ambeth Kumar and Dr.M.Ramakrishan(2011), "Footprint Based Recognition System" in the month of April for the International Journal Communication in Computer and Information System (CCIS) Journal (Springer) Volume 147, Part 3, 358-367, DOI: 10.1007/978-3-642-20573-6_63, April 2011

[18] V.D.Ambeth Kumar (2017), "Efficient Routing for Low Rate Wireless Network a Novel Approach", International Journal of Image Mining, Vol. 2, Nos. 3/4, 2017, 2017

[19] V.D.Ambeth Kumar, V.D.Ashok Kumar, Dr.S.Malathi and P.Jagaeedesh, (2014) "Intruder Identification using Footprint Recognition with PCA and SVM Classifiers" for the International Journal of Advanced Materials Research Vols.1345, PP 984-985 (2014) pp 1345-1349. [DOI:10.4028/www.scientific.net/AMR.984-985.1345]

[20] V.D.Ambeth Kumar, G.Gokul, S.Malathi, K.Vengatesan, D.Elangovan, B.Chitra, "Implementation Of The Pulse Rhythemic Rate For The Efficient Diagonising Of The Heart Beat", ", Healthcare Technology Letters (IET) 2019 Apr 17;6(2):48-52.

[21] S.V. Ruphitha et.al, " Management of Major Postpartum Haemorrhage by using Zigbee protocol - A Review “, 2021 6th International Conference on Inventive Computation Technologies (ICICT) (DOI: 10.1109/ICICT50816.2021.9358757 )

[22] M.Ramakrishan et.al., "Footprint Based Recognition System" in the month of April for the International Journal Communication in Computer and Information System (CCIS) Journal (Springer) Volume 147, Part 3, 358-367, DOI: 10.1007/978-3-642-20573-6 63, April 2011

[23] M. Indhumathi et.al , "Healthcare Management of Major Cardiovascular Disease-A review", 2021 6th International Conference on Inventive Computation Technologies (ICICT), (DOI: 10.1109/ICICT50816.2021.9358519)

[24] V.D.Ambeth Kumar, Dr.S.Malathi, V.D.Ashok Kumar (2015) "Performance Improvement Using an Automation System for Segmentation of Multiple Parametric Features Based on Human Footprint" for the Journal of Electrical Engineering \& Technology (JEET), vol. 10, no. 4, pp.1815-1821 , 2015. [http://dx.doi.org/10.5370/JEET.2015.10.4.1815]

[25] Dr.V.D.Ambeth Kumar, S.Vaishali and B.Shweta (2015) "Basic Study of the Human Foot" for the Journal of Biomedical and Pharmacology, Volume 08 No. 01 Page No. 435-444 Jun, 2015.[DOI: $10.13005 / \mathrm{bpj} / 632$.

[26] V.D.Ambeth Kumar and M.Ramakrishan (2013) "A Comparative Study of Fuzzy Evolutionary Techniques for Footprint Recognition and Performance Improvement using Wavelet based Fuzzy Neural Network" for the International Journal of Computer Applications in Technology, Vol.48, No.2,pp.95 - 105, [DOI: ttp://dx.doi.org/10.1504/IJCAT.2013.056016]

[27] Navadeepika K.M.R., Ambeth Kumar V.D. (2021) Enhancement in Braille Systems-A Survey", Advances in Intelligent Systems and Computing, vol 1257. Springer, Singapore. https://doi.org/10.1007/978-981-15-7907-3_10.

[28] V.D. Ambeth Kumar and S. Sharmila, "An Clinical Study on Finding Hypothermia and Postpartum Haemorrhage Using Temperature”, International Journal of Psychosocial Rehabilitation, Vol. 24, Issue 05, pp: 2624- 2629, 2020.

[29] Navadeepika.K.M.R, Dr.V.D.Ambeth Kumar, "Online Conversion of Tamil Language into Tactile for Visual Impaired People”, International Journal of Advanced Science and Technology Vol. 29, No. 5, pp. $3401-3407,(2020)$.

[30] A. Vignesh, T. YokeshSelvan, Ganesh Krishnan, Arjun N. Sasikumar, V. D. Ambeth Kumar, "Efficient Student Profession Prediction Using XGBoost Algorithm", Lecture Notes on Data Engineering and Communications Technologies, Volume 35, pp 140-148, 2020.

[31] . HariKeshav, H. Divakar, R. Gokul, G. Senthil Kumar, V. D. Ambeth Kumar, "Real Time Categorical Availability of Blood Units in a Blood Bank Using IoT", Lecture Notes on Data Engineering and Communications Technologies, Volume 35, pp 503-510, 2020.

[32] R. Christina Rini, V. D. Ambeth Kumar, "Cost Effective Decision Support Product for Finding the Postpartum Haemorrhage", Lecture Notes on Data Engineering and Communications Technologies, Volume 35, pp 649-656, 2020. 